\title{
Optimizing the signal-to-noise ratio for direct-detection BOTDA
}

Sheng Wang, Zhisheng Yang, Marcelo A. Soto, Luc Thévenaz

Sheng Wang, Zhisheng Yang, Marcelo A. Soto, Luc Thévenaz, "Optimizing the signal-to-noise ratio for direct-detection BOTDA," Proc. SPIE 11199, Seventh European Workshop on Optical Fibre Sensors, 111992C (28 August 2019); doi: $10.1117 / 12.2540930$

Event: Seventh European Workshop on Optical Fibre Sensors, 2019, Limassol, Cyprus 


\title{
Optimizing the signal-to-noise ratio for direct-detection BOTDA
}

\author{
Sheng Wang, ${ }^{\mathrm{a}, \mathrm{b}}$, Zhisheng Yang ${ }^{* a}$, Marcelo A. Soto ${ }^{\mathrm{a}, \mathrm{c}}$ and Luc Thévenaz \\ ${ }^{a}$ EPFL, Group for Fibre Optics, SCI STI LT Station 11, 1015 Lausanne, Switzerland; beijing \\ University of Posts and Telecommunications, 100876 Beijing, China; ${ }^{\mathrm{c} D e p a r t m e n t ~ o f ~ E l e c t r o n i c ~}$ \\ Engineering, Universidad Técnica Federico Santa María, 2390123 Valparaíso, Chile \\ *Email: zhisheng.yang@epfl.ch
}

\begin{abstract}
The signal-to-noise ratio (SNR) of the measurement for direct-detection Brillouin optical time-domain analyzers is modelled and experimentally validated, with and without the use of optical pre-amplification. Results indicate that preamplification associated with a good-quality photo-detector improves considerably the actual SNR, with only $1.5 \mathrm{~dB}$ penalty compared to the ideal shot noise limit.
\end{abstract}

Keywords: Optical fiber sensor, stimulated Brillouin scattering

\section{INTRODUCTION}

Brillouin optical time-domain analysis (BOTDA) has stimulated much research efforts over a couple of decades for distributed temperature and strain measurements ${ }^{1}$. Exploiting stimulated Brillouin scattering (SBS) through the interaction of a pump and a probe, BOTDA shows a larger signal response compared to other distributed fiber sensors that normally utilize spontaneous scatterings. Therefore, BOTDA is widely considered as a good candidate for long-distance (>25 km) sensing. Former studies in literature conclude that the overall performance of BOTDA is scaled and traded-off by the signal-to-noise ratio $(\mathrm{SNR})^{2}$, which constitutes the key parameter that ultimately defines the measurement quality. To improve the SNR, various methods have been proposed to either enhance the signals by optimizing optical schemes ${ }^{3}$, or reduce the noise employing post-processing ${ }^{4}$. Despite the abundance of those sophisticated methods, the optimization of the detection scheme has been poorly discussed in the community and there is currently no explicit guidelines regarding how to select a proper detector and detection scheme for a given scenario. In addition, as the noise in long-distance BOTDA systems is mainly determined by the detection stage, it is of crucial necessity to quantify the impact of detection schemes on the trace SNR. It must be noted that the peculiarities of the BOTDA signal (small informative modulation on top of a strong continuous background) make a simple extrapolation from optimized schemes in optical communication irrelevant.

In this paper, we model the impact of direct detection on SNR of traditional BOTDA, with and without the use of optical pre-amplification right in front of the detection stage. The study is performed as a function of different probe powers reaching the detection stage, regardless of the detailed sensor layout and range. Models are experimentally validated using two distinct detectors with 3-dB bandwidths of $75 \mathrm{MHz}$ and $350 \mathrm{MHz}$, respectively. Results indicate that the use of optical pre-amplification, associated to a good PD design, significantly improves the SNR, regardless of the added optical noise.

\section{DIRECT DETECTION}

In a conventional BOTDA, the converted photocurrent $I_{S}$ through the photodiode presents a large but non-informative DC component $I_{D C}$ and an AC signal $I_{A C}$ that carries the essence of the distributed Brillouin information. For meter-scale spatial resolutions and long distance ranges, $I_{A C}$ can be very well approximated by $g I_{D C}$, where $g<10 \%$ represents the Brillouin gain experienced by the probe and depends on the position along the fiber and the pump-probe frequency difference. Based on this small-gain approximation, sources of detection noise depending on the optical power (e.g., shot noise) are considered as mainly attributed to $I_{D C}$. For a measurement using the simplest direct detection, the noise is essentially made of the thermal noise $\sigma_{t h}^{2}$ and the shot noise $\sigma_{s h}^{2}$, so that the SNR can be expressed as:

$$
S N R_{\mathrm{Di}}=\frac{I_{s}}{\sigma_{N}}=\frac{I_{A C}}{\sqrt{\sigma_{t h}^{2}+\sigma_{s h}^{2}}}=\frac{g I_{D C}}{\sqrt{\frac{4 k_{B} T B_{e}}{R_{L}}+2 q I_{D C} B_{e}}}
$$

Seventh European Workshop on Optical Fibre Sensors, edited by Kyriacos Kalli, Gilberto Brambilla, Sinead O'Keeffe Proc. of SPIE Vol. 11199, 111992C · (c) 2019 SPIE · CCC code: 0277-786X/19/\$21 · doi: 10.1117/12.2540930 
where the Boltzmann constant $k_{B}$, the working temperature $T$ and the electron charge $q$ are fundamental/environmental quantities offering no degree of freedom, while the photo-receiver resistor $R_{L}$ and the noise equivalent bandwidth (NEB) $B_{e}$ depend on the PD design. It should be noted that some PDs include a post-amplification stage that increases the thermal noise, so that the term $\sigma_{t h}^{2}$ in Eq. (1) is not representative enough in most practical cases and the total thermal noise must actually be measured experimentally. On the contrary, the term $\sigma_{s h}^{2}$ is always valid for predicting the shot noise. Thus, Eq. (1) can be conveniently modified as:

$$
S N R_{\mathrm{Di}}=\frac{g I_{D C}}{\sqrt{\sigma_{T}^{2} B_{e}+2 q I_{D C} B_{e}}}
$$

where $\sigma_{T}^{2}$ is the power spectral density $\left(A^{2} / \mathrm{Hz}\right)$ of the total measured thermal noise (i.e. including the noise added by postamplification). Eq. (2) indicates that, the SNR is determined by both $I_{D C}$ and $g$ for a given PD. Practically, $I_{D C}$ depends on the BOTDA set-up and the sensing range, while $g$ depends on the spatial resolution, pump power, pump-probe frequency difference and fiber position. For the sake of simplicity and without loss of generality, in this study $g$ is considered as a scaling factor, and the SNR is investigated as a function of $I_{D C}$ considering a probe optical power (reaching the receiver) ranging from $-50 \mathrm{dBm}$ to $-5 \mathrm{dBm}$, which covers most of the BOTDA sensing scenarios. Two common commercially available PDs with 3-dB bandwidths $\left(B_{c}\right)$ of $75 \mathrm{MHz}$ and $350 \mathrm{MHz}$, showing the specifications listed in Table 1, are used to evaluate and verify the model. It can be found that both PDs include post-amplifiers because both $\sigma_{T}^{2}$ do not match the thermal noise expression in Eq. (1); however, PDB435 is better performing (less impaired by the post-amplifier in PD), as its $\sigma_{T}^{2}$ is only 7 times larger than that of PDB425 while its saturation power is $14 \mathrm{~dB}$ ( 25 times) higher.

Table 1. Parameters of two PDs

\begin{tabular}{|c|c|c|c|c|c|}
\hline Type & $\begin{array}{c}B_{c} \\
(\mathrm{MHz})\end{array}$ & $\begin{array}{c}B_{e} \\
(\mathrm{MHz})\end{array}$ & $\begin{array}{c}\text { Conversion Gain } \\
(\mathrm{V} / \mathrm{A})\end{array}$ & $\begin{array}{c}\text { Saturation power } \\
(\mathrm{dBm})\end{array}$ & $\begin{array}{c}\sigma_{T}^{2} \\
\left(\mathrm{~A}^{2} / \mathrm{Hz}\right)\end{array}$ \\
\hline PDB425 & 75 & 120 & 125000 & -18 & $3.499 \mathrm{e}-23$ \\
\hline PDB435 & 350 & 600 & 5000 & -4 & $2.535 \mathrm{e}-22$ \\
\hline
\end{tabular}

First, to validate the theoretical expression of the noise term (denominator of Eq. (2)), the calculated results versus different $I_{D C}$ for both PDs are illustrated in Fig. 1(a) and (b), which are in good agreements with measurements. It can be observed that both PDs are essentially thermal noise dominated, and the shot noise only contributes slightly (3\% for PDB425 and $11 \%$ for PD435) to the total noise close to the saturation power levels.
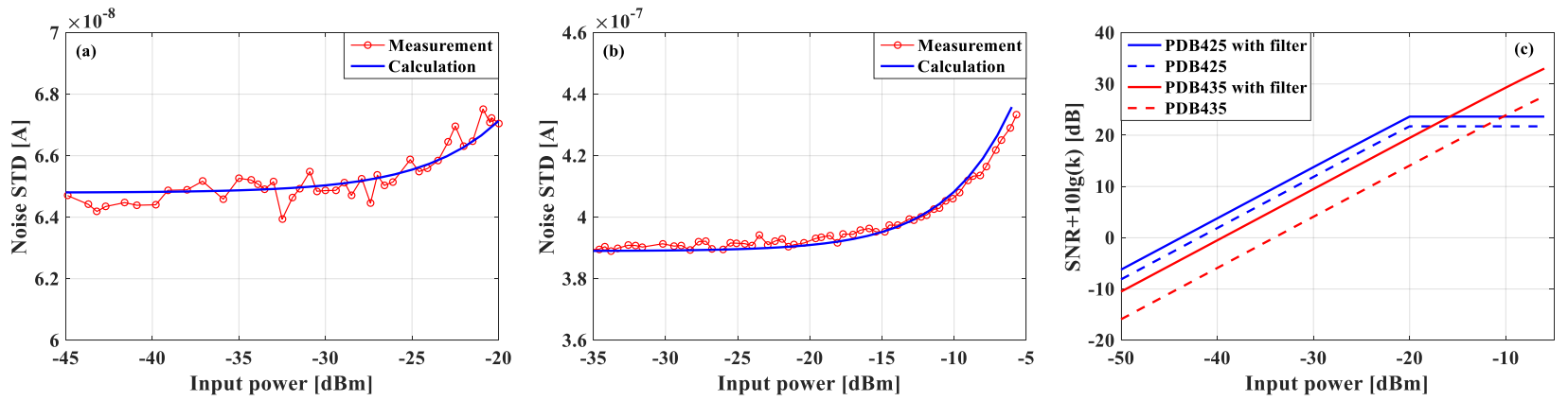

Figure 1. Measured detection noise as a function of input power using (a) PDB425; (b) PDB435; (c) the behavior of SNRs as a function of input power with and without digital filtering.

This noise validation allows for further predicting the overall SNR for any $g$ value, as shown by the dashed lines in Fig. 1(c). It can be found that PDB425 always shows $8 \mathrm{~dB}$ better SNR than PDB435 before saturating, due to the narrower $B_{e}$ and the smaller $\sigma_{T}^{2}$. As $I_{D C}$ increases, the input power on PDB425 has to be limited, thus making the SNR unchanged. On the other hand, the SNR of PDB435 continues increasing and becomes larger than that of PDB425 at input powers larger than $-12 \mathrm{dBm}$. It is worth mentioning that the SNR behavior may be different after applying post-processing (e.g., digital Gaussian fileting ${ }^{4}$ ), since the effective bandwidth $B_{e}$ for both PDs can be optimized to match the signal bandwidth $B_{s}$ that is only determined by the spatial resolution. This way the trace SNR could be improved by a factor of $\sqrt{B_{e} / B_{s}}$, as shown by the solid lines in Fig. 1(c) for an exemplified case of $B_{S}=50 \mathrm{MHz}$ corresponding to a $2 \mathrm{~m}$ spatial resolution. PDB435 can reach a larger improvement due to the larger original $B_{e}$, so that the SNR crossing point for these two PDs is shifted down to $-16 \mathrm{dBm}$. 


\section{DIRECT DETECTION WITH EDFA}

From Eq. (2) and Fig. 1(c), it can be found that in any case the highest SNR is obtained when the input signal power is near the saturation level. However, it is not possible to reach this optimal condition when the sensing distance is long, and therefore a pre-amplifier (e.g., an EDFA) is often inserted in front of the PD to enhance the SNR. This optical amplification increases the detected optical signal by a factor given by the EDFA gain $G$ (i.e., $I_{G}=G I_{D C}$ ), at the expense of introducing the amplified spontaneous emission (ASE) noise that is added on the signal photocurrent. This EDFA-induced noise mainly leads to signal-ASE beating noise $I_{s-s p}=2 F_{N} q(G-1) I_{G} B_{e}$ and ASE-ASE beating noise $I_{s p-s p}=F_{N}^{2} q^{2}(G-1)^{2} B_{o} B_{e}{ }^{5}$, where $F_{N}$ is the noise figure of the EDFA (ideally $F_{N}=2$ ) and $B_{o}$ is the optical bandwidth. In most standard BOTDA implementations, a narrowband filter is placed after the EDFA to select one frequency sideband of the probe and to reduce the ASE noise. This leads to a fairly narrow optical noise bandwidth $B_{o}$, so that the contribution of $I_{s p-s p}$ can be safely neglected in all well-designed BOTDA implementations. This way the SNR in the case of direct detection with EDFA can be expressed by simply adding the contribution of $I_{s-s p}$ into Eq. (2):

$$
S N R_{\mathrm{Di}-\mathrm{EDFA}}=\frac{g I_{G}}{\sqrt{\sigma_{T}^{2} B_{e}+2 q I_{G} B_{e}+2 F_{N} q(G-1) I_{G} B_{e}}}
$$

The noise term, i.e., the denominator of Eq. (3), is experimentally validated for both PDs this way: a continuous wave $(\mathrm{CW})$ representing the DC probe ranging from $-50 \mathrm{dBm}$ to $-5 \mathrm{dBm}$ is launched into an EDFA followed by a narrow bandwidth $(6 \mathrm{GHz})$ optical filter. The filter output signal is first sent to an optical spectrum analyzer (OSA) to measure the amplified signal power, so that the value of $G$ is determined as shown by the blue dots in Fig. 2(a). As expected, $G$ gradually decreases as the EDFA input power increases, due to the use of the constant-current mode in the EDFA pumping and its subsequent saturation. Note that in practice the amplified signal may saturate the PD for large $G$, so that a tunable attenuator is usually inserted between the filter and the PD to set the signal power as high as possible but just below the PD saturation level (i.e., $I_{G}=I_{\text {sat }}$ ). Because of such additional attenuation, $G$ is reduced to be equal to the ratio between the PD saturation power and the input power of the EDFA. So the actual $G$ values used in Eq. (3) for both PDs have to be rescaled, as shown by the red and green curves in Fig. 2(a).
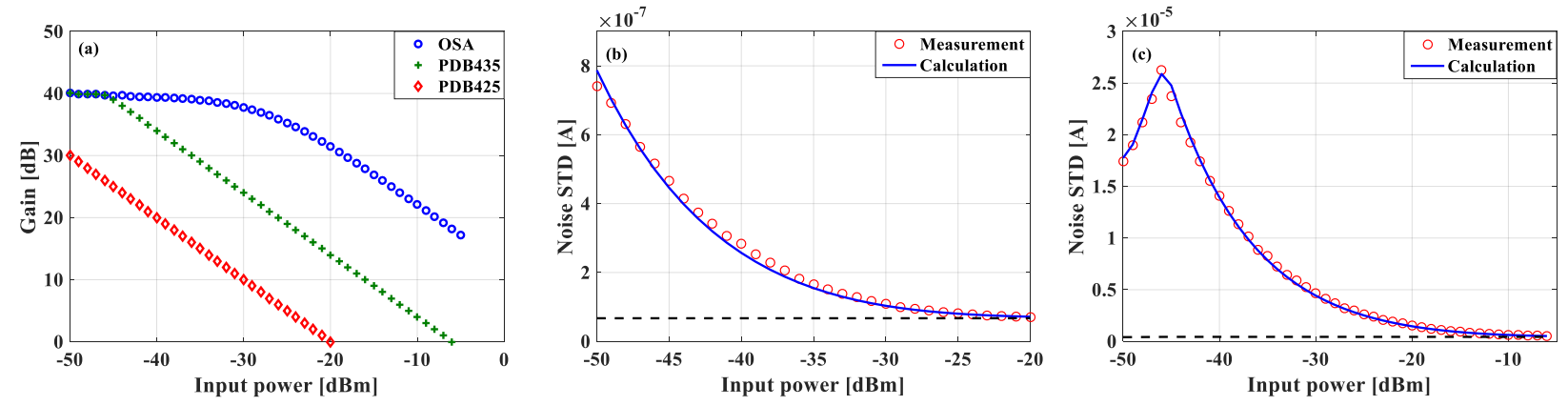

Figure 2. (a) The gain of EDFA as a function of different input power; Measured detection noise for pre-amplified direct detection using (b) PDB425; (c) PDB435;

Knowing the practical gain value $G$ (i.e., considering the additional attenuation) as a function of the input EDFA power enables to further estimate the noise. Fig. 2(b) and (c) show the calculated and measured noises for PDB425 and PDB435, respectively, where the black dashed lines stand for the contribution of the thermal noise. It can be seen that for both PDs the noise is dominated by $I_{s-s p}$ when the input power is low. When the input power turns higher, the noise decreases and becomes dominated essentially by the thermal noise at powers approaching the saturation level. It is also observed that the noise grows as the input power increases from $-50 \mathrm{dBm}$ to $-46 \mathrm{dBm}$ when using PDB435. This is due to the fact that the EDFA output does not saturate the PD in this regime, so that $G$ keeps constant and the curve trend strictly follows the increase of the input power. Theoretical curves and experiments are in good agreements, validating the noise model expressed by Eq. (3) and making the SNR estimation possible. A general case without post-processing is firstly studied, as shown in Fig. 3(a). It indicates that the SNR for pre-amplified direct detection (red and blue solid lines) is always better than that of the direct detection with no amplification (red and blue dashed lines), and the SNR improvement decreases with the increase of input power. Note that at low input powers, PDB425 leads to better SNR than PDB435, however this situations is inverted at powers higher than $-23 \mathrm{dBm}$ due to the synthesis impact of $G, B_{e}$ and $\sigma_{T}^{2}$. 
The impact of digital filtering on the SNR is investigated in Fig. 3(b), for the case of $B_{s}=50 \mathrm{MHz}$, corresponding to a $2 \mathrm{~m}$ spatial resolution. The figure shows that PDB435 after EDFA always leads to a similar or better SNR compared to PDB425. More specifically, both PDs show similar SNR performance when the EDFA input power is less than $-35 \mathrm{dBm}$, since the noise is highly dominated by signal-ASE beating $I_{s-s p}$, so that the SNR expression can be simplified as:

$$
S N R_{\mathrm{Di-EDFA}}=g \sqrt{\frac{I_{D C}}{2 F_{N} q B_{S}}}=\sqrt{\frac{1}{F_{N}}} S N R_{\mathrm{no}-\mathrm{EDFA}}^{\text {shot }}
$$

which is independent of any PD specifications and is represented by the black dashed line in Fig. 3(b). For a higher EDFA input power, the shot noise and thermal noise become non-negligible; thus, the difference between the real SNR and the one estimated by Eq. (4) (black dashed line) gets larger. Since the PDB435 is better performing (less impaired by postamplification in PD), the difference is smaller than that of PDB425. From Eq. (4) it can be also observed that the preamplified direct-detection scheme only exhibits a SNR penalty of $1.5 \mathrm{~dB}\left(\sqrt{F_{N}}\right)$ with respect to the case of shot-noise limited (green dashed line in Fig. 3), which might eventually be reached using much more sophisticated detection schemes, for instance based on coherent detection.
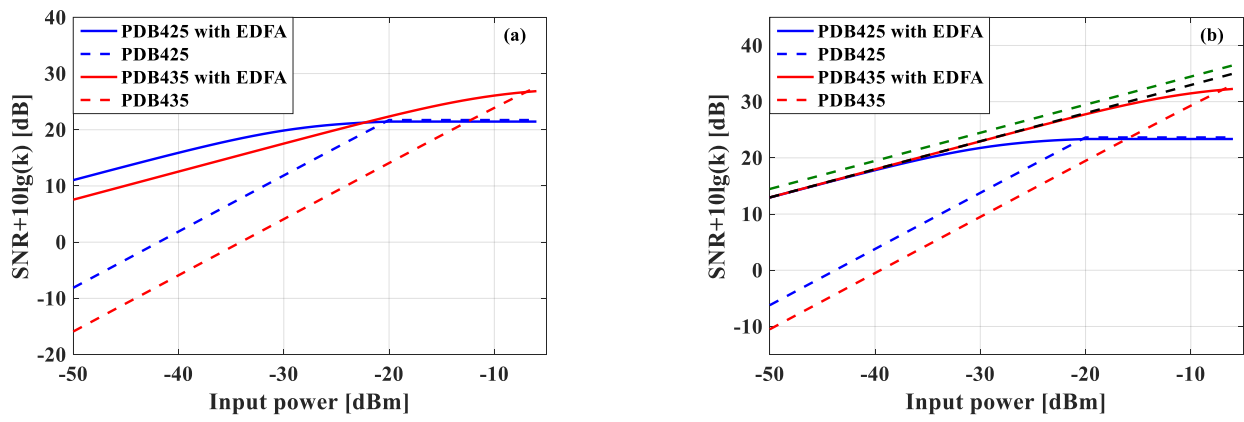

Figure 3. The behavior of SNRs as a function of different probe power (a) without and (b) with digital filtering.

\section{CONCLUSION}

Results highlight that the shot-noise limit is close to be reached at any probe power by direct detection with prior optical amplification, using a selected pre-amplifier with a low noise figure, a well-designed PD and a digital filtering to match noise and signal bandwidths. Such optimized SNR can be readily estimated using Eq. (4), which offers an analytical tool to evaluate the BOTDA performance in different scenarios. Regarding the simplicity of the configuration compared to for instance coherent detection, with no local oscillator and polarization-diversity processing, it indicates that direct detection remains a very effective solution for BOTDA that is difficult to be practically outperformed.

Sheng Wang acknowledges the China Scholarship Council (CSC) for supporting his stay at EPFL.

\section{REFERENCES}

[1] Motil, A., Bergman, A. and Tur, M., "State of the art of Brillouin fiber-optic distributed sensing," Optics \& Laser Technology 78, part A, 81-103 (2016).

[2] Soto, M. A., and Thévenaz L., "Modeling and evaluating the performance of Brillouin distributed optical fiber sensors," Opt. Express 21(25), 31347-31366 (2013).

[3] Soto, M. A., [Distributed Brillouin Sensing: Time-Domain Techniques], Springer Nature Publishers, Singapore 45-51 (2018).

[4] Zaslawski, S., et al., "Impact of Fitting and Digital Filtering on Signal-to-Noise Ratio and Brillouin Frequency Shift Uncertainty of BOTDA Measurements" 26th International Conference on Optical Fiber Sensors, ThE27 (2018).

[5] Jacobs, I., "Dependence of optical amplifier noise figure on relative-intensity-noise," J. Lightwave Technol. 13(7), 1461-1465 (1995). 